\title{
Some compact and non-compact embedding theorems for the function spaces defined by fractional Fourier transform
}

\author{
Erdem Toksoy (i), Ayşe Sandıkçı* (D) \\ Department of Mathematics, Faculty of Art and Sciences, Ondokuz Mayıs University, Samsun 55139, \\ Turkey
}

\begin{abstract}
The fractional Fourier transform is a generalization of the classical Fourier transform through an angular parameter $\alpha$. This transform uses in quantum optics and quantum wave field reconstruction, also its application provides solving some differrential equations which arise in quantum mechanics. The aim of this work is to discuss compact and non-compact embeddings between the spaces $A_{\alpha, p}^{w, \omega}\left(\mathbb{R}^{d}\right)$ which are the set of functions in $L_{w}^{1}\left(\mathbb{R}^{d}\right)$ whose fractional Fourier transform are in $L_{\omega}^{p}\left(\mathbb{R}^{d}\right)$. Moreover, some relevant counterexamples are indicated.
\end{abstract}

Mathematics Subject Classification (2020). 42A38, 43A15, 47B07

Keywords. fractional Fourier transform, weighted Lebesgue spaces, compact embedding

\section{Introduction}

Throughout this article, we study on $\mathbb{R}^{d}$. For any function $f: \mathbb{R}^{d} \rightarrow \mathbb{C}$, the translation and modulation operators are defined as $T_{y} f(t)=f(t-y)$ and $M_{w} f(t)=e^{i w t} f(t)$ for all $y, w \in \mathbb{R}^{d}$, respectively [18]. $C_{C}\left(\mathbb{R}^{d}\right)$ denotes the space of continuous complex function on $\mathbb{R}^{d}$ whose support is compact, and also $C_{0}\left(\mathbb{R}^{d}\right)$ denotes the space of continuous complex functions on $\mathbb{R}^{d}$ which vanish at infinity [17]. Besides we write the Lebesgue space $\left(L^{p}\left(\mathbb{R}^{d}\right),\|\cdot\|_{p}\right)$, for $1 \leq p<\infty$. A weight (Beurling weight) function $w$ on $\mathbb{R}^{d}$ is a measurable and locally bounded function that satisfies $w(x) \geq 1$ and $w(x+y) \leq$ $w(x) w(y)$ (submultiplicative, [11]) for all $x, y \in \mathbb{R}^{d}$. Troughout the article we consider Beurling weights. We define, for $1 \leq p<\infty$,

$$
L_{w}^{p}\left(\mathbb{R}^{d}\right)=\left\{f \mid f w \in L^{p}\left(\mathbb{R}^{d}\right)\right\} .
$$

It is well known that $L_{w}^{p}\left(\mathbb{R}^{d}\right)$ is a Banach space under the norm $\|f\|_{p, w}=\|f w\|_{p}[16]$.

\footnotetext{
*Corresponding Author.

Email addresses: erdem.toksoy@omu.edu.tr (E. Toksoy), ayses@omu.edu.tr (A. Sandıkçı)

Received: 16.09.2020; Accepted: 13.06.2021
} 
Let $w_{1}$ and $w_{2}$ be two weight functions. We say that $w_{1} \prec w_{2}$ if there exists $c>0$, such that $w_{1}(x) \leq c w_{2}(x)$ for all $x \in \mathbb{R}^{d},[9]$. We define the Fourier transform $\widehat{f}$ (or $\mathcal{F} f$ ) of a function $f \in L^{1}(\mathbb{R})$ as

$$
\widehat{f}(w)=\mathcal{F} f(w)=\frac{1}{\sqrt{2 \pi}} \int_{-\infty}^{+\infty} f(t) e^{-i w t} d t .
$$

The fractional Fourier transform was first studied by Wiener [20] in 1929. In [20], this new transformation has been viewed as a generalization of the Fourier transform. The importance of this transformation has been understood by Namias' work [14]. Namias used the fractional Fourier transform as a tool for solving ordinary and partial differential equations arising in quantum mechanics. McBride and Kerr [13], using the results of Namias, described this transformation in the Schwartz space and examined its properties. This transform has many applications in signal processing, optics, filtering and timefrequency analysis $[1,2,6,15]$. The fractional Fourier transform is a generalization of the Fourier transform through an angle parameter $\alpha$ and can be considered as a rotation by an angle $\alpha$ in the time-frequency plane. The fractional Fourier transform with angle $\alpha$ of a function $f \in L^{1}(\mathbb{R})$ is defined by

$$
\mathcal{F}_{\alpha} f(u)=\int_{-\infty}^{+\infty} K_{\alpha}(u, t) f(t) d t
$$

where,

$$
K_{\alpha}(u, t)= \begin{cases}\sqrt{\frac{1-i \cot \alpha}{2 \pi}} e^{i\left(\frac{u^{2}+t^{2}}{2}\right) \cot \alpha-i u t \operatorname{cosec} \alpha} & \text { if } \alpha \text { is not multiple of } \pi \\ \delta(t-u), & \text { if } \alpha=2 k \pi, k \in \mathbb{Z} \\ \delta(t+u), & \text { if } \alpha=(2 k+1) \pi, k \in \mathbb{Z}\end{cases}
$$

and $\delta$, Dirac delta function. The fractional Fourier transform with $\alpha=\frac{\pi}{2}$ corresponds to the Fourier transform, $[2-4,14,15,21]$.

The fractional Fourier transform can be extended for higher dimensions as [4]:

$$
\left(\mathcal{F}_{\alpha_{1}, \ldots, \alpha_{n}} f\right)\left(u_{1}, \ldots, u_{n}\right)=\int_{-\infty}^{+\infty} \ldots \int_{-\infty}^{+\infty} K_{\alpha_{1}, \ldots, \alpha_{n}}\left(u_{1}, \ldots, u_{n} ; t_{1}, \ldots, t_{n}\right) f\left(t_{1}, \ldots, t_{n}\right) d t_{1} \ldots d t_{n}
$$

or shortly

$$
\mathcal{F}_{\alpha} f(u)=\int_{-\infty}^{+\infty} \ldots \int_{-\infty}^{+\infty} K_{\alpha}(u, t) f(t) d t
$$

where

$$
K_{\alpha}(u, t)=K_{\alpha_{1}, \ldots, \alpha_{n}}\left(u_{1}, \ldots, u_{n} ; t_{1}, \ldots, t_{n}\right)=K_{\alpha_{1}}\left(u_{1}, t_{1}\right) K_{\alpha_{2}}\left(u_{2}, t_{2}\right) \ldots K_{\alpha_{n}}\left(u_{n}, t_{n}\right) .
$$

Let $w$ and $\omega$ be weight functions on $\mathbb{R}^{d}$ and $1 \leq p<\infty$. The space $A_{\alpha, p}^{w, \omega}\left(\mathbb{R}^{d}\right)$ consists of all $f \in L_{w}^{1}\left(\mathbb{R}^{d}\right)$ such that $\mathcal{F}_{\alpha} f \in L_{\omega}^{p}\left(\mathbb{R}^{d}\right)$. The linear space $A_{\alpha, p}^{w, \omega}\left(\mathbb{R}^{d}\right)$ is a Banach space under the norm:

$$
\|f\|_{A_{\alpha, p}^{w, \omega}}=\|f\|_{1, w}+\left\|\mathcal{F}_{\alpha} f\right\|_{p, \omega} .
$$

The space $A_{\alpha, p}^{w, \omega}\left(\mathbb{R}^{d}\right)$ is translation and modulation invariant space [19]. The family of all translation and modulation invariant spaces, studied recently in [5]. In this work we discuss compact and non-compact embeddings between the spaces $A_{\alpha, p}^{w, \omega}\left(\mathbb{R}^{d}\right)$. General compactness criteria for function spaces are given in [7] or more general in [8]. 
Let $\alpha=\left(\alpha_{1}, \alpha_{2}, \ldots, \alpha_{d}\right)$, where $\alpha_{i}=2 k \pi$ for each index $i$ with $1 \leq i \leq d$ and $k \in \mathbb{Z}$. Then by definition of fractional Fourier transform, $\mathcal{F}_{\alpha} f(u)=f(u)$ for all $u \in \mathbb{R}^{d}$. Thus we have

$$
A_{\alpha, p}^{w, \omega}\left(\mathbb{R}^{d}\right)=\left\{f \in L_{w}^{1}\left(\mathbb{R}^{d}\right) \mid f \in L_{\omega}^{p}\left(\mathbb{R}^{d}\right)\right\}=L_{w}^{1}\left(\mathbb{R}^{d}\right) \cap L_{\omega}^{p}\left(\mathbb{R}^{d}\right) .
$$

Let $\alpha=\left(\alpha_{1}, \alpha_{2}, \ldots, \alpha_{d}\right)$, where $\alpha_{i}=(2 k+1) \pi$ for each index $i$ with $1 \leq i \leq d$ and $k \in \mathbb{Z}$. Then by definition of fractional Fourier transform, $\mathcal{F}_{\alpha} f(u)=f(-u)$ for all $u \in \mathbb{R}^{d}$. We may assume without loss of generality that $\omega$ symmetric, i.e., satisfy $\omega(x)=\omega(-x)$ for all $x \in \mathbb{R}^{d}$. Again, we obtain (1.1). Troughout this study, unless otherwise indicated, we get $\alpha=\left(\alpha_{1}, \alpha_{2}, \ldots, \alpha_{d}\right)$, where $\alpha_{i} \neq k \pi$ for each index $i$ with $1 \leq i \leq d$ and $k \in \mathbb{Z}$. In this paper we extend the results of [12] to the fractional Fourier transform. An angle parameter $\alpha$ provides us a new aspect of results that have been established in [12]. Additionally, we give some examples of why a necessary condition cannot be provided.

\section{Some compact and non-compact embedding theorems for the function spaces $A_{\alpha, p}^{w, \omega}\left(\mathbb{R}^{d}\right)$}

We begin with the following basic findings that will be used as a tool to show the inclusion properties and non-compact embeddings of the spaces $A_{\alpha, p}^{w, \omega}\left(\mathbb{R}^{d}\right)$.

Proposition 2.1. Let $\alpha=\left(\alpha_{1}, \alpha_{2}, \ldots, \alpha_{d}\right)$, where $\alpha_{i} \neq k \pi$ for each index $i$ with $1 \leq i \leq d$ and $k \in \mathbb{Z}$. Let $w$ and $\omega$ be weight functions on $\mathbb{R}^{d}$ and $g \in A_{\alpha, p}^{w, \omega}\left(\mathbb{R}^{d}\right)$. Then

$$
\left\|T_{y} M_{b} g\right\|_{A_{\alpha, p}^{w, \omega}} \leq w(y)\|g\|_{1, w}+\left\|\mathcal{F}_{\alpha} g\right\|_{p, \omega}
$$

and so $T_{y} M_{b} g \in A_{\alpha, p}^{w, \omega}\left(\mathbb{R}^{d}\right)$, where $b=\left(-y_{1} \cot \alpha_{1}, \ldots,-y_{d} \cot \alpha_{d}\right)$ for all $y=\left(y_{1}, \ldots, y_{d}\right) \in$ $\mathbb{R}^{d}$.

Proof. Let $b=\left(-y_{1} \cot \alpha_{1}, \ldots,-y_{d} \cot \alpha_{d}\right)$ for all $y=\left(y_{1}, \ldots, y_{d}\right) \in \mathbb{R}^{d}$ and let $g \in$ $A_{\alpha, p}^{w, \omega}\left(\mathbb{R}^{d}\right)$. Then, $g \in L_{w}^{1}\left(\mathbb{R}^{d}\right)$ and $\mathcal{F}_{\alpha} g \in L_{\omega}^{p}\left(\mathbb{R}^{d}\right)$. We may write by $[10$, Proposition 1.7]

$$
\begin{aligned}
\left\|T_{y} M_{b} g\right\|_{1, w}=\int_{\mathbb{R}^{d}}|g(x-y)|\left|e^{i(x-y) b}\right| w(x) d x & =\left\|T_{y} g\right\|_{1, w} \\
& \leq w(y)\|g\|_{1, w} .
\end{aligned}
$$

Also, we have

$$
\begin{aligned}
\left\|\mathcal{F}_{\alpha}\left(T_{y} M_{b} g\right)\right\|_{p, \omega}^{p} & =\int_{\mathbb{R}^{d}}\left|\mathcal{F}_{\alpha}\left(T_{y} M_{b} g\right)(u)\right|^{p} \omega^{p}(u) d u \\
= & \left|\prod_{j=1}^{d} \sqrt{\frac{1-i \cot \alpha_{j}}{2 \pi}}\right|^{p} \\
& \times \int_{\mathbb{R}^{d}}\left|\int_{\mathbb{R}^{d}}\left(T_{y} M_{b} g\right)(t) e^{\sum_{j=1}^{d}\left(\frac{i}{2}\left(u_{j}^{2}+t_{j}^{2}\right) \cot \alpha_{j}-i u_{j} t_{j} \operatorname{cosec} \alpha_{j}\right)} d t\right|^{p} \omega^{p}(u) d u \\
= & \mid \prod_{j=1}^{d}{\sqrt{\frac{1-i \cot \alpha_{j}}{2 \pi}}}^{p}
\end{aligned}
$$




$$
\begin{aligned}
& \times \int_{\mathbb{R}^{d}} \mid \int_{\mathbb{R}^{d}} g(t-y) e^{\sum_{j=1}^{d} i\left(t_{j}-y_{j}\right) b_{j}} \\
& \times e^{\sum_{j=1}^{d}\left(\frac{i}{2}\left(u_{j}^{2}+t_{j}^{2}\right) \cot \alpha_{j}-i u_{j} t_{j} \operatorname{cosec} \alpha_{j}\right)} d t||^{p} \omega^{p}(u) d u .
\end{aligned}
$$

By substitution $t-y=l$, we get

$$
\begin{aligned}
& \left\|\mathcal{F}_{\alpha}\left(T_{y} M_{b} g\right)\right\|_{p, \omega}^{p}=\left|\prod_{j=1}^{d} \sqrt{\frac{1-i \cot \alpha_{j}}{2 \pi}}\right|^{p} \\
& \times \int_{\mathbb{R}^{d}} \mid \int_{\mathbb{R}^{d}} g(l) e^{\sum_{j=1}^{d}\left(\frac{i}{2}\left(u_{j}^{2}+\left(l_{j}+y_{j}\right)^{2}\right) \cot \alpha_{j}-i u_{j}\left(l_{j}+y_{j}\right) \operatorname{cosec} \alpha_{j}\right)} \\
& \times\left. e^{\sum_{j=1}^{d}-i l_{j} y_{j} \cot \alpha_{j}} d l\right|^{p} \omega^{p}(u) d u \\
& =\left|\prod_{j=1}^{d} \sqrt{\frac{1-i \cot \alpha_{j}}{2 \pi}}\right|_{\mathbb{R}^{d}}^{p}\left|e^{\sum_{j=1}^{d}\left(\frac{i}{2} y_{j}^{2} \cot \alpha_{j}-i u_{j} y_{j} \operatorname{cosec} \alpha_{j}\right)}\right|^{p} \\
& \times\left|\int_{\mathbb{R}^{d}} g(l) e^{\sum_{j=1}^{d}\left(\frac{i}{2}\left(u_{j}^{2}+l_{j}^{2}\right) \cot \alpha_{j}-i u_{j} l_{j} \operatorname{cosec} \alpha_{j}\right)} d l\right|^{p} \omega^{p}(u) d u \\
& =\int_{\mathbb{R}^{d}}\left|\mathcal{F}_{\alpha} g(u)\right|^{p} \omega^{p}(u) d u \\
& =\left\|\mathcal{F}_{\alpha} g\right\|_{p, \omega}^{p} .
\end{aligned}
$$

Therefore, we obtain inequality (2.1). This means that $T_{y} M_{b} g \in A_{\alpha, p}^{w, \omega}\left(\mathbb{R}^{d}\right)$.

The following proposition will be needed to show the inclusion properties of the space $A_{\alpha, p}^{w, \omega}\left(\mathbb{R}^{d}\right)$.

Proposition 2.2. Let $\alpha=\left(\alpha_{1}, \alpha_{2}, \ldots, \alpha_{d}\right)$, where $\alpha_{i} \neq k \pi$ for each index $i$ with $1 \leq i \leq d$ and $k \in \mathbb{Z}$. Let $w$ and $\omega$ be weight functions on $\mathbb{R}^{d}$. For every $0 \neq g \in A_{\alpha, p}^{w, \omega}\left(\mathbb{R}^{d}\right)$ there exists constants $c(g), c\left(\mathcal{F}_{\alpha} g\right)>0$ such that

$$
c(g) w(y) \leq\left\|T_{y} M_{b} g\right\|_{A_{\alpha, p}^{w, \omega}} \leq w(y)\|g\|_{A_{\alpha, p}^{w, \omega}}
$$

and

$$
c\left(\mathcal{F}_{\alpha} g\right) \omega(a) \leq\left\|M_{z} g\right\|_{A_{\alpha, p}^{w, \omega}} \leq \omega(a)\|g\|_{A_{\alpha, p}^{w, \omega}}
$$

where $b=\left(-y_{1} \cot \alpha_{1}, \ldots,-y_{d} \cot \alpha_{d}\right)$ and $a=\left(z_{1} \sin \alpha_{1}, \ldots, z_{d} \sin \alpha_{d}\right)$ for all $y=$ $\left(y_{1}, \ldots, y_{d}\right), z=\left(z_{1}, \ldots, z_{d}\right) \in \mathbb{R}^{d}$, respectively.

Proof. Let us take $b=\left(-y_{1} \cot \alpha_{1}, \ldots,-y_{d} \cot \alpha_{d}\right)$ and $a=\left(z_{1} \sin \alpha_{1}, \ldots, z_{d} \sin \alpha_{d}\right)$ for all $y=\left(y_{1}, \ldots, y_{d}\right), z=\left(z_{1}, \ldots, z_{d}\right) \in \mathbb{R}^{d}$, respectively. Let $0 \neq g \in A_{\alpha, p}^{w, \omega}\left(\mathbb{R}^{d}\right)$. Thus 
$g \in L_{w}^{1}\left(\mathbb{R}^{d}\right)$ and $\mathcal{F}_{\alpha} g \in L_{\omega}^{p}\left(\mathbb{R}^{d}\right)$. By [10, Proposition 1.7], there exists $c(g)>0$ such that

$$
c(g) w(y) \leq\left\|T_{y} g\right\|_{1, w} \leq w(y)\|g\|_{1, w} .
$$

Using (2.2), (2.3) and (2.6), we obtain

$$
\begin{aligned}
c(g) w(y) & \leq\left\|T_{y} g\right\|_{1, w} \leq\left\|T_{y} M_{b} g\right\|_{1, w}+\left\|\mathcal{F}_{\alpha}\left(T_{y} M_{b} g\right)\right\|_{p, \omega} \\
& \leq w(y)\|g\|_{1, w}+w(y)\left\|\mathcal{F}_{\alpha} g\right\|_{p, \omega}=w(y)\|g\|_{A_{\alpha, p}^{w, \omega}} .
\end{aligned}
$$

This means inequality (2.4) holds.

By Proposition 3 (2) in [19], we have

$$
\left\|\mathcal{F}_{\alpha}\left(M_{z} g\right)\right\|_{p, \omega}=\left\|T_{a} \mathcal{F}_{\alpha} g\right\|_{p, \omega} .
$$

Again, by [10, Proposition 1.7], there exists $c\left(\mathcal{F}_{\alpha} g\right)>0$ such that

$$
c\left(\mathcal{F}_{\alpha} g\right) \omega(a) \leq\left\|T_{a} \mathcal{F}_{\alpha} g\right\|_{p, \omega} \leq \omega(a)\left\|\mathcal{F}_{\alpha} g\right\|_{p, \omega} .
$$

Combining (2.7), (2.8) and the equality $\left\|M_{z} g\right\|_{1, w}=\|g\|_{1, w}$, we get

$$
\begin{aligned}
c\left(\mathcal{F}_{\alpha} g\right) \omega(a) & \leq\left\|T_{a} \mathcal{F}_{\alpha} g\right\|_{p, \omega} \leq\left\|M_{z} g\right\|_{1, w}+\left\|\mathcal{F}_{\alpha}\left(M_{z} g\right)\right\|_{p, \omega} \\
& \leq \omega(a)\|g\|_{1, w}+\omega(a)\left\|\mathcal{F}_{\alpha} g\right\|_{p, \omega}=\omega(a)\|g\|_{A_{\alpha, p}^{w, \omega}} .
\end{aligned}
$$

The proof of the following lemma is very similiar to the proof of Lemma 11 in [19], and therefore we omit the details.

Lemma 2.3. Let $\alpha=\left(\alpha_{1}, \alpha_{2}, \ldots, \alpha_{d}\right)$, where $\alpha_{i} \neq k \pi$ for each index $i$ with $1 \leq i \leq d$ and $k \in \mathbb{Z}$. Let $w, \omega$ and $v$ be weight functions on $\mathbb{R}^{d}$. If $A_{\alpha, p}^{w, \omega}\left(\mathbb{R}^{d}\right) \subset L_{v}^{1}\left(\mathbb{R}^{d}\right)$, then $A_{\alpha, p}^{w, \omega}\left(\mathbb{R}^{d}\right)$ is a Banach space under the norm $|\|g\||=\|g\|_{A_{\alpha, p}^{w, \omega}}+\|g\|_{1, v}$.

Theorem 2.4. Let $\alpha=\left(\alpha_{1}, \alpha_{2}, \ldots, \alpha_{d}\right)$, where $\alpha_{i} \neq k \pi$ for each index $i$ with $1 \leq i \leq d$ and $k \in \mathbb{Z}$. Let $w, \omega$ and $v$ be weight functions on $\mathbb{R}^{d}$. Then $A_{\alpha, p}^{w, \omega}\left(\mathbb{R}^{d}\right) \subset L_{v}^{1}\left(\mathbb{R}^{d}\right)$ if and only if $v \prec w$.

Proof. Assume that $v \prec w$. Hence, there exists $c_{1}>0$ such that $v(x) \leq c_{1} w(x)$ for all $x \in \mathbb{R}^{d}$. Let $g \in A_{\alpha, p}^{w, \omega}\left(\mathbb{R}^{d}\right)$. Thus $g \in L_{w}^{1}\left(\mathbb{R}^{d}\right)$ and $\mathcal{F}_{\alpha} g \in L_{\omega}^{p}\left(\mathbb{R}^{d}\right)$. We write

$$
\|g v\|_{1} \leq c_{1}\|g w\|_{1}
$$

and then

$$
\|g\|_{1, v} \leq c_{1}\|g\|_{1, w} .
$$

This implies

$$
\|g\|_{1, v} \leq c_{1}\|g\|_{1, w}+c_{1}\left\|\mathcal{F}_{\alpha} g\right\|_{p, \omega}=c_{1}\|g\|_{A_{\alpha, p}^{w, \omega}}<\infty .
$$

Therefore, $A_{\alpha, p}^{w, \omega}\left(\mathbb{R}^{d}\right) \subset L_{v}^{1}\left(\mathbb{R}^{d}\right)$.

Conversely, assume that $A_{\alpha, p}^{w, \omega}\left(\mathbb{R}^{d}\right) \subset L_{v}^{1}\left(\mathbb{R}^{d}\right)$. Let us take $b=$ $\left(-y_{1} \cot \alpha_{1}, \ldots,-y_{d} \cot \alpha_{d}\right)$ for all $y=\left(y_{1}, \ldots, y_{d}\right) \in \mathbb{R}^{d}$. By Proposition 2.2, there exists constants $c_{2}, c_{3}>0$ such that

$$
c_{2} w(y) \leq\left\|T_{y} M_{b} g\right\|_{A_{\alpha, p}^{w, \omega}} \leq c_{3} w(y) .
$$

By [9, Lemma 2.2] and (2.2), there exists constants $c_{4}, c_{5}>0$ such that

$$
c_{4} v(y) \leq\left\|T_{y} g\right\|_{1, v}=\left\|T_{y} M_{b} g\right\|_{1, v} \leq c_{5} v(y) .
$$


From Lemma 2.3 the space $A_{\alpha, p}^{w, \omega}\left(\mathbb{R}^{d}\right)$ is a Banach space under the norm $|\|g\||$ for all $g \in A_{\alpha, p}^{w, \omega}\left(\mathbb{R}^{d}\right)$. Then the closed graph theorem implies the existence of a constant $c_{6}>0$ such that $\|g\|_{1, v} \leq c_{6}\|g\|_{A_{\alpha, p}^{w, \omega}}$ for all $g \in A_{\alpha, p}^{w, \omega}\left(\mathbb{R}^{d}\right)$. Since $T_{y} M_{b} g \in A_{\alpha, p}^{w, \omega}\left(\mathbb{R}^{d}\right)$, we get

$$
\left\|T_{y} M_{b} g\right\|_{1, v} \leq c_{6}\left\|T_{y} M_{b} g\right\|_{A_{\alpha, p}^{w, \omega}}
$$

Thus, combining (2.9), (2.10) and (2.11), we obtain

$$
c_{4} v(y) \leq\left\|T_{y} g\right\|_{1, v}=\left\|T_{y} M_{b} g\right\|_{1, v} \leq c_{6}\left\|T_{y} M_{b} g\right\|_{A_{\alpha, p}^{w, \omega}} \leq c_{6} c_{3} w(y) .
$$

Let $c=\frac{c_{6} c_{3}}{c_{4}}$. Then we have $v(y) \leq c w(y)$ for all $y \in \mathbb{R}^{d}$.

The following theorem is the extension of Theorem 12 in [19].

Theorem 2.5. Let $\alpha=\left(\alpha_{1}, \alpha_{2}, \ldots, \alpha_{d}\right)$, where $\alpha_{i} \neq k \pi$ for each index $i$ with $1 \leq i \leq d$ and $k \in \mathbb{Z}$. Let $w_{1}, w_{2}, \omega_{1}$ and $\omega_{2}$ be weight functions on $\mathbb{R}^{d}$. Then $A_{\alpha, p}^{w_{1}, \omega_{1}}\left(\mathbb{R}^{d}\right) \subset A_{\alpha, p}^{w_{2}, \omega_{2}}\left(\mathbb{R}^{d}\right)$ if and only if $w_{2} \prec w_{1}$ and $\omega_{2} \prec \omega_{1}$.

Proof. First of all, suppose that $w_{2} \prec w_{1}$ and $\omega_{2} \prec \omega_{1}$. Then it is clear that $A_{\alpha, p}^{w_{1}, \omega_{1}}\left(\mathbb{R}^{d}\right) \subset$ $A_{\alpha, p}^{w_{2}, \omega_{2}}\left(\mathbb{R}^{d}\right)$ by $[19$, Proposition 13$]$.

Now, assume that $A_{\alpha, p}^{w_{1}, \omega_{1}}\left(\mathbb{R}^{d}\right) \subset A_{\alpha, p}^{w_{2}, \omega_{2}}\left(\mathbb{R}^{d}\right)$. By Proposition 2.2, there exist constants $c_{1}, c_{2}, c_{3}, c_{4}>0$ such that

$$
c_{1} w_{1}(y) \leq\left\|T_{y} M_{b} g\right\|_{A_{\alpha, p}^{w_{1}, \omega_{1}}} \leq c_{2} w_{1}(y)
$$

and

$$
c_{3} w_{2}(y) \leq\left\|T_{y} M_{b} g\right\|_{A_{\alpha, p}^{w_{2}, \omega_{2}}} \leq c_{4} w_{2}(y)
$$

where $b=\left(-y_{1} \cot \alpha_{1}, \ldots,-y_{d} \cot \alpha_{d}\right)$ for all $y=\left(y_{1}, \ldots, y_{d}\right) \in \mathbb{R}^{d}$. According to Lemma 11 in [19], the space $A_{\alpha, p}^{w_{1}, \omega_{1}}\left(\mathbb{R}^{d}\right)$ is a Banach space under the norm

$$
|\|g\||=\|g\|_{A_{\alpha, p}^{w_{1}, \omega_{1}}}+\|g\|_{A_{\alpha, p}^{w_{2}, \omega_{2}}}
$$

for all $g \in A_{\alpha, p}^{w_{1}, \omega_{1}}\left(\mathbb{R}^{d}\right)$. Therefore, $w_{2} \prec w_{1}$ follows from the closed graph theorem and applying the same technique in the proof of the Theorem 2.4. Again, by Proposition 2.2, there exist constants $c_{5}, c_{6}, c_{7}, c_{8}>0$ such that

$$
c_{5} \omega_{1}(a) \leq\left\|M_{z} g\right\|_{A_{\alpha, p}^{w_{1}, \omega_{1}}} \leq c_{6} \omega_{1}(a)
$$

and

$$
c_{7} \omega_{2}(a) \leq\left\|M_{z} g\right\|_{A_{\alpha, p}^{w_{2}, \omega_{2}}} \leq c_{8} \omega_{2}(a)
$$

where $a=\left(z_{1} \sin \alpha_{1}, \ldots, z_{d} \sin \alpha_{d}\right)$ for all $z=\left(z_{1}, \ldots, z_{d}\right) \in \mathbb{R}^{d}$. Then the closed graph theorem implies the existence of a constant $c_{9}>0$ such that $\|g\|_{A_{\alpha, p}^{w_{2}, \omega_{2}} \leq} \leq c_{9}\|g\|_{A_{\alpha, p}^{w_{1}, \omega_{1}}}$ for all $g \in A_{\alpha, p}^{w_{1}, \omega_{1}}\left(\mathbb{R}^{d}\right)$. Since $M_{z} g \in A_{\alpha, p}^{w_{1}, \omega_{1}}\left(\mathbb{R}^{d}\right)$, we get

$$
\left\|M_{z} g\right\|_{A_{\alpha, p}^{w_{2}, \omega_{2}}} \leq c_{9}\left\|M_{z} g\right\|_{A_{\alpha, p}^{w_{1}, \omega_{1}}}
$$

Thus, combining (2.15), (2.16) and (2.17), we obtain

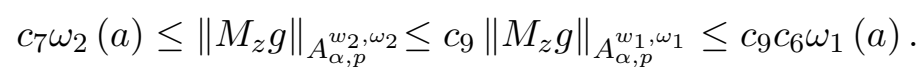

Let $c=\frac{c_{9} c_{6}}{c_{7}}$. Then we have $\omega_{2}(a) \leq c \omega_{1}(a)$ for all $a \in \mathbb{R}^{d}$. 
Theorem 2.6. Let $\alpha=\left(\alpha_{1}, \alpha_{2}, \ldots, \alpha_{d}\right)$, where $\alpha_{i} \neq k \pi$ for each index $i$ with $1 \leq i \leq d$ and $k \in \mathbb{Z}$. Let $1 \leq p<\infty$ and $w, \omega$ and $v$ be weight functions on $\mathbb{R}^{d}$. If $v \prec w$ and the function $\omega$ is bounded then $A_{\alpha, p}^{w, \omega}\left(\mathbb{R}^{d}\right) \hookrightarrow L_{v}^{1}\left(\mathbb{R}^{d}\right)$ is not compact.

Proof. Since $v \prec w$, then there exists $c_{1}>0$ such that $v(x) \leq c_{1} w(x)$ for all $x \in \mathbb{R}^{d}$ and also $A_{\alpha, p}^{w, \omega}\left(\mathbb{R}^{d}\right) \subset L_{v}^{1}\left(\mathbb{R}^{d}\right)$ by Theorem 2.4. Besides the unit map $I$ from $A_{\alpha, p}^{w, \omega}\left(\mathbb{R}^{d}\right)$ into $L_{v}^{1}\left(\mathbb{R}^{d}\right)$ is bounded

$$
\|I(f)\|_{1, v}=\|f\|_{1, v} \leq c_{1}\|f\|_{1, w} \leq c_{1}\|f\|_{A_{\alpha, p}^{w, \omega}}
$$

for all $f \in A_{\alpha, p}^{w, \omega}\left(\mathbb{R}^{d}\right)$.

Let $g \in C_{C}\left(\mathbb{R}^{d}\right) \cap A_{\alpha, p}^{w, \omega}\left(\mathbb{R}^{d}\right)$ be a fixed arbitrary function. We take sequence $\left(t_{n}\right)_{n \in \mathbb{N}}$ in $\mathbb{R}^{d}$ such that $t_{n} \rightarrow \infty$ as $n \rightarrow \infty$ and define a sequence $\left(g_{n}\right)_{n \in \mathbb{N}}$ by $g_{n}=M_{t_{n}} g$. From the hypothesis, there exists $c_{2} \geq 1$ such that $\omega(x) \leq c_{2}$ for all $x \in \mathbb{R}^{d}$. Then we write

$$
\left\|g_{n}\right\|_{A_{\alpha, p}^{w, \omega}}=\left\|M_{t_{n}} g\right\|_{A_{\alpha, p}^{w, \omega}} \leq\|g\|_{1, w}+c_{2}\left\|\mathcal{F}_{\alpha} g\right\|_{p, \omega} \leq c_{2}\|g\|_{A_{\alpha, p}^{w, \omega}}
$$

Hence, the sequence $\left(g_{n}\right)_{n \in \mathbb{N}}$ is bounded in $A_{\alpha, p}^{w, \omega}\left(\mathbb{R}^{d}\right)$. Now, we will show that there is no subsequence of $\left(g_{n}\right)_{n \in \mathbb{N}}$ that is convergent in $L_{v}^{1}\left(\mathbb{R}^{d}\right)$. Let $h \in C_{C}\left(\mathbb{R}^{d}\right)$ be given. We get

$$
\begin{aligned}
\left|\int_{\mathbb{R}^{d}} g_{n}(x) h(x) d x\right| & \leq \int_{\mathbb{R}^{d}}\left|M_{t_{n}} g(x)\right||h(x)| d x \\
& \leq\|h\|_{\infty}\|g\|_{1} \\
& =\|h\|_{\infty}\left\|T_{t_{n}} g\right\|_{1} .
\end{aligned}
$$

Since $g \in C_{C}\left(\mathbb{R}^{d}\right) \subset C_{0}\left(\mathbb{R}^{d}\right), T_{t_{n}} g \rightarrow 0$ as $n \rightarrow \infty$ and then there exists $N \in \mathbb{N}$ such that $\operatorname{suppg}\left(x-t_{n}\right)=\varnothing$ for all $n \geq N$ and all $x \in \mathbb{R}^{d}$. On the other hand we get $\operatorname{suppg}\left(x-t_{j}\right)=K_{j}$ where $j=1,2, \ldots, N-1$. Let $K=K_{1} \cup K_{2} \cup \ldots \cup K_{N-1}$. Then, using (2.19) we write

$$
\left|\int_{\mathbb{R}^{d}} g_{n}(x) h(x) d x\right| \leq\|h\|_{\infty} \int_{K}\left|T_{t_{n}} g(x)\right| d x .
$$

Also we have

$$
\left|T_{t_{n}} g(x)\right|=\left|g\left(x-t_{n}\right)\right| \leq \sup _{x-t_{n} \in \mathbb{R}^{d}}\left|g\left(x-t_{n}\right)\right|=\|g\|_{\infty}
$$

for all $n \in \mathbb{N}$. Then by bounded converge theorem and using (2.20), (2.21) we obtain

$$
\int_{\mathbb{R}^{d}} g_{n}(x) h(x) d x \rightarrow 0
$$

as $n \rightarrow \infty$. Assume that $\left(g_{n}\right)_{n \in \mathbb{N}}$ converges to $f \neq 0$ in $L_{v}^{1}\left(\mathbb{R}^{d}\right)$. Thus we may write

$$
\left|\int_{\mathbb{R}^{d}}\left(g_{n}-f\right)(x) h(x) d x\right| \leq \int_{\mathbb{R}^{d}}\left|\left(g_{n}-f\right)(x)\right||h(x)| d x \leq\|h\|_{\infty}\left\|g_{n}-f\right\|_{1, v} .
$$

Since $\left\|g_{n}-f\right\|_{1, v} \rightarrow 0$ as $n \rightarrow \infty$, then we have

$$
\int_{\mathbb{R}^{d}}\left(g_{n}-f\right)(x) h(x) d x \rightarrow 0
$$


as $n \rightarrow \infty$. Hence by using (2.22), we obtain

$$
\int_{\mathbb{R}^{d}} f(x) h(x) d x=0
$$

for all $h \in C_{C}\left(\mathbb{R}^{d}\right)$. Then $f=0$ almost everywehere. This is a contradiction. Therefore only possible limit of $\left(g_{n}\right)_{n \in \mathbb{N}}$ in $L_{v}^{1}\left(\mathbb{R}^{d}\right)$ is zero. It is known that there exist $c_{3}(g), c_{4}>0$ such that

$$
c_{3}(g) v(x) \leq\left\|T_{x} g\right\|_{1, v} \leq c_{4} v(x)
$$

for all $g \in L_{v}^{1}\left(\mathbb{R}^{d}\right)$ and $x \in \mathbb{R}^{d},[9]$. Therefore, we obtain

$$
\left\|g_{n}\right\|_{1, v}=\left\|M_{t_{n}} g\right\|_{1, v}=\|g\|_{1, v} \geq \frac{\left\|T_{t_{n}} g\right\|_{1, v}}{v\left(t_{n}\right)} \geq \frac{c_{3}(g) v\left(t_{n}\right)}{v\left(t_{n}\right)}=c_{3}(g) .
$$

Then $\left(g_{n}\right)_{n \in \mathbb{N}}$ does not converge to zero and also it is impossible to find convergent subsequence of $\left(g_{n}\right)_{n \in \mathbb{N}}$ in $L_{v}^{1}\left(\mathbb{R}^{d}\right)$. In that case, the embedding $I$ is not compact.

Let us give an example that the converse of the above theorem is not correct.

Example 2.7. Let $\alpha=\left(\alpha_{1}, \alpha_{2}, \ldots, \alpha_{d}\right)$, where $\alpha_{i} \neq k \pi$ for each index $i$ with $1 \leq i \leq d$ and $k \in \mathbb{Z}$. Let $w, \omega$ and $v$ be weight functions on $\mathbb{R}^{d}$ such that $v(x)=w(x)=(1+\|x\|)^{2}$ and $\omega(x)=1+\|x\|$, for all $x \in \mathbb{R}^{d}$. It is easy to see that these functions are weight functions. Also let us take $\alpha=\left(\alpha_{1}, \alpha_{2}, \ldots, \alpha_{d}\right)$, where $\alpha_{1}=\alpha_{2}=\ldots=\alpha_{d}$. Then the embedding $I$ from $A_{\alpha, p}^{w, \omega}\left(\mathbb{R}^{d}\right)$ into $L_{v}^{1}\left(\mathbb{R}^{d}\right)$ is not compact and the function $\omega$ is not bounded. Indeed, $v \prec w$ then $A_{\alpha, p}^{w, \omega}\left(\mathbb{R}^{d}\right) \subset L_{v}^{1}\left(\mathbb{R}^{d}\right)$ and also the unit map $I$ is continuous from $A_{\alpha, p}^{w, \omega}\left(\mathbb{R}^{d}\right)$ into $L_{v}^{1}\left(\mathbb{R}^{d}\right)$. Let us take any function $g \in A_{\alpha, p}^{w, \omega}\left(\mathbb{R}^{d}\right)$ and fix it. Besides, we take sequence $\left(t_{n}\right)_{n \in \mathbb{N}}$ in $\mathbb{R}^{d}$ such that $t_{n} \rightarrow \infty$ as $n \rightarrow \infty$ and define a sequence $\left(g_{n}\right)_{n \in \mathbb{N}}$ by $g_{n}=\frac{T_{t_{n}} g}{w\left(t_{n}\right)}=\frac{T_{t_{n} g}}{\left(1+\left\|t_{n}\right\|\right)^{2}}$. Therefore, we have

$$
\left\|g_{n}\right\|_{A_{\alpha, p}^{w, \omega}}=\left\|\frac{T_{t_{n}} g}{w\left(t_{n}\right)}\right\|_{A_{\alpha, p}^{w, \omega}}=\frac{1}{w\left(t_{n}\right)}\left\|T_{t_{n}} g\right\|_{A_{\alpha, p}^{w, \omega}} .
$$

Also we write

$$
\omega(x)=1+\|x\| \leq(1+\|x\|)^{2}=w(x) .
$$

Moreover we have the inequality

$$
\left\|T_{x} g\right\|_{A_{\alpha, p}^{w, \omega}} \leq w(x)\|g\|_{1, w}+\omega\left(x \cos \alpha_{1}\right)\left\|\mathcal{F}_{\alpha} g\right\|_{p, \omega}
$$

for all $x \in \mathbb{R}^{d}$, by Theorem 4 in [19]. Then, by using (2.26) we get

$$
\begin{aligned}
\left\|T_{x} g\right\|_{A_{\alpha, p}^{w, \omega}} & \leq w(x)\|g\|_{1, w}+\omega\left(x \cos \alpha_{1}\right)\left\|\mathcal{F}_{\alpha} g\right\|_{p, \omega} \\
& =w(x)\|g\|_{1, w}+\left(1+\left|\cos \alpha_{1}\right|\|x\|\right)\left\|\mathcal{F}_{\alpha} g\right\|_{p, \omega} \\
& \leq w(x)\|g\|_{1, w}+(1+\|x\|)\left\|\mathcal{F}_{\alpha} g\right\|_{p, \omega} \\
& \leq w(x)\|g\|_{A_{\alpha, p}^{w, \omega}} .
\end{aligned}
$$

Thus we have

$$
\left\|g_{n}\right\|_{A_{\alpha, p}^{w, \omega}}=\left\|\frac{T_{t_{n}} g}{w\left(t_{n}\right)}\right\|_{A_{\alpha, p}^{w, \omega}} \leq \frac{w\left(t_{n}\right)}{w\left(t_{n}\right)}\|g\|_{A_{\alpha, p}^{w, \omega}}=\|g\|_{A_{\alpha, p}^{w, \omega}}
$$


Hence, the sequence $\left(g_{n}\right)_{n \in \mathbb{N}}$ is bounded in $A_{\alpha, p}^{w, \omega}\left(\mathbb{R}^{d}\right)$. Let $h \in C_{C}\left(\mathbb{R}^{d}\right)$ be given. We write

$$
\begin{aligned}
\left|\int_{\mathbb{R}^{d}} g_{n}(x) h(x) d x\right| \leq & \frac{1}{w\left(t_{n}\right)} \int_{\mathbb{R}^{d}}\left|T_{t_{n}} g(x)\right||h(x)| d x \\
& \leq \frac{1}{w\left(t_{n}\right)}\|h\|_{\infty}\|g\|_{1} .
\end{aligned}
$$
obtain

Since $w(x)$ tends to infinity as $x \rightarrow \infty, w\left(t_{n}\right)$ tends to infinity as $n \rightarrow \infty$. Then we

$$
\int_{\mathbb{R}^{d}} g_{n}(x) h(x) d x \rightarrow 0
$$

as $n \rightarrow \infty$. After that, by using the same technique in the proof of Theorem 2.6, we obtain that the embedding $I$ is not compact. On the other hand, $\omega(x) \rightarrow \infty$ as $x \rightarrow \infty$, and then the function $\omega$ is not bounded.

Theorem 2.8. Let $\alpha=\left(\alpha_{1}, \alpha_{2}, \ldots, \alpha_{d}\right)$, where $\alpha_{i} \neq k \pi$ for each index $i$ with $1 \leq i \leq d$ and $k \in \mathbb{Z}$. Let $1 \leq p<\infty$ and $w, \omega$ and $v$ be weight functions on $\mathbb{R}^{d}$. If $v \prec w$ and function $\frac{v(x)}{w(x)}$ does not tend to zero for $x \rightarrow \infty$, then the embedding

$$
I: A_{\alpha, p}^{w, \omega}\left(\mathbb{R}^{d}\right) \hookrightarrow L_{v}^{1}\left(\mathbb{R}^{d}\right)
$$

is never compact.

Proof. Since $v \prec w$, Theorem 2.4 and the proof of Theorem 2.6 implies that $A_{\alpha, p}^{w, w}\left(\mathbb{R}^{d}\right) \subset$ $L_{v}^{1}\left(\mathbb{R}^{d}\right)$ and continuity of the unit map $I$, respectively.

First of all, suppose that $w(x) \rightarrow \infty$ as $x \rightarrow \infty$. Let us take any fixed function $g \in A_{\alpha, p}^{w, \omega}\left(\mathbb{R}^{d}\right)$. Also we take sequence $\left(t_{n}\right)_{n \in \mathbb{N}}$ in $\mathbb{R}^{d}$ such that $t_{n} \rightarrow \infty$ as $n \rightarrow \infty$. Then we define a sequence $\left(b_{n}\right)_{n \in \mathbb{N}}$ in $\mathbb{R}^{d}$ such that $b_{n j}=-t_{n j} \cot \alpha_{j}$. To show that the mapping $I: A_{\alpha, p}^{w, \omega}\left(\mathbb{R}^{d}\right) \rightarrow L_{v}^{1}\left(\mathbb{R}^{d}\right)$ is never compact, define a sequence $\left(g_{n}\right)_{n \in \mathbb{N}}$ by $g_{n}=\frac{T_{t_{n}} M_{b_{n}} g}{w\left(t_{n}\right)}$. Therefore, we have

$$
\left\|g_{n}\right\|_{A_{\alpha, p}^{w, \omega}}=\left\|\frac{T_{t_{n}} M_{b_{n}} g}{w\left(t_{n}\right)}\right\|_{A_{\alpha, p}^{w, \omega}}=\frac{1}{w\left(t_{n}\right)}\left\|T_{t_{n}} M_{b_{n}} g\right\|_{A_{\alpha, p}^{w, \omega}} .
$$

By using (2.1) we write

$$
\left\|g_{n}\right\|_{A_{\alpha, p}^{w, \omega}} \leq \frac{w\left(t_{n}\right)}{w\left(t_{n}\right)}\|g\|_{A_{\alpha, p}^{w, \omega}}=\|g\|_{A_{\alpha, p}^{w, \omega}}
$$

Hence, the sequence $\left(g_{n}\right)_{n \in \mathbb{N}}$ is bounded in $A_{\alpha, p}^{w, \omega}\left(\mathbb{R}^{d}\right)$. Now, we will show that there wouldn't be a subsequence of $\left(g_{n}\right)_{n \in \mathbb{N}}$ which is convergent in $L_{v}^{1}\left(\mathbb{R}^{d}\right)$. We have

$$
\begin{aligned}
\left|\int_{\mathbb{R}^{d}} g_{n}(x) h(x) d x\right| & \leq \frac{1}{w\left(t_{n}\right)} \int_{\mathbb{R}^{d}}\left|T_{t_{n}} M_{b_{n}} g(x)\right||h(x)| d x \\
& \leq \frac{1}{w\left(t_{n}\right)}\|h\|_{\infty}\|g\|_{1}
\end{aligned}
$$

for all $h \in C_{C}\left(\mathbb{R}^{d}\right)$. Since $w\left(t_{n}\right) \rightarrow \infty$ as $n \rightarrow \infty$, then by using inequality (2.31) we obtain

$$
\int_{\mathbb{R}^{d}} g_{n}(x) h(x) d x \rightarrow 0
$$


as $n \rightarrow \infty$. Moreover, by the inequality

$$
\begin{aligned}
\left|\int_{\mathbb{R}^{d}} g_{n}(x) h(x) d x\right| & \leq \int_{\mathbb{R}^{d}}\left|g_{n}(x)\right||h(x)| d x \\
& \leq \sup _{x \in \mathbb{R}^{d}}|h(x)| \int_{\mathbb{R}^{d}}\left|g_{n}(x)\right| d x \\
& \leq\|h\|_{\infty}\left\|g_{n}\right\|_{1, v}
\end{aligned}
$$

and using the same technique in the proof of Theorem 2.6, we say that only possible limit of $\left(g_{n}\right)_{n \in \mathbb{N}}$ in $L_{v}^{1}\left(\mathbb{R}^{d}\right)$ is zero. Also, using (2.23) and (2.2), there exists $c>0$ such that

$$
\left\|g_{n}\right\|_{1, v}=\frac{1}{w\left(t_{n}\right)}\left\|T_{t_{n}} M_{b_{n}} g\right\|_{1, v}=\frac{1}{w\left(t_{n}\right)}\left\|T_{t_{n}} g\right\|_{1, v} \geq c \frac{v\left(t_{n}\right)}{w\left(t_{n}\right)}
$$

Since the function $\frac{v\left(t_{n}\right)}{w\left(t_{n}\right)}$ does not tend to zero for $n \rightarrow \infty$ and then there exists $\delta>0$ such that $\frac{v\left(t_{n}\right)}{w\left(t_{n}\right)} \geq \delta$ as $n \rightarrow \infty$. Then by using (2.33) we have

$$
\left\|g_{n}\right\|_{1, v} \geq \frac{v\left(t_{n}\right)}{w\left(t_{n}\right)} c \geq c \delta
$$

Hence, $\left(g_{n}\right)_{n \in \mathbb{N}}$ does not converge to zero and also it is impossible to find convergent subsequence of $\left(g_{n}\right)_{n \in \mathbb{N}}$ in $L_{v}^{1}\left(\mathbb{R}^{d}\right)$. Consequently, the embedding $I: A_{\alpha, p}^{w, \omega}\left(\mathbb{R}^{d}\right) \rightarrow L_{v}^{1}\left(\mathbb{R}^{d}\right)$ is never compact.

Suppose that $w$ is constant or bounded function. Since $v \prec w$, the function $\frac{v(x)}{w(x)}$ is constant or bounded and then doesn't tend to zero as $x \rightarrow \infty$. Let us take any fixed function $g \in C_{C}\left(\mathbb{R}^{d}\right) \cap A_{\alpha, p}^{w, \omega}\left(\mathbb{R}^{d}\right)$. Besides we take a sequence $\left(t_{n}\right)_{n \in \mathbb{N}}$ in $\mathbb{R}^{d}$ such that $t_{n} \rightarrow \infty$ as $n \rightarrow \infty$. Then we define a sequence $\left(g_{n}\right)_{n \in \mathbb{N}}$ by $g_{n}=\frac{T_{t_{n}} M_{b_{n}} g}{w\left(t_{n}\right)}$, where $\left(b_{n}\right)_{n \in \mathbb{N}}$ is a sequence in $\mathbb{R}^{d}$ such that $b_{n j}=-t_{n j} \cot \alpha_{j}$. Thus, the sequence $\left(g_{n}\right)_{n \in \mathbb{N}}$ is bounded in $A_{\alpha, p}^{w, \omega}\left(\mathbb{R}^{d}\right)$ by $(2.30)$. Let $h \in C_{C}\left(\mathbb{R}^{d}\right)$ be given. We write

$$
\begin{aligned}
\left|\int_{\mathbb{R}^{d}} g_{n}(x) h(x) d x\right| & \leq \frac{1}{w\left(t_{n}\right)} \int_{\mathbb{R}^{d}}\left|T_{t_{n}} M_{b_{n}} g(x)\right||h(x)| d x \\
& \leq \frac{1}{w\left(t_{n}\right)}\|h\|_{\infty} \int_{\mathbb{R}^{d}}\left|T_{t_{n}} g(x)\right| d x .
\end{aligned}
$$

Since $g \in C_{C}\left(\mathbb{R}^{d}\right) \subset C_{0}\left(\mathbb{R}^{d}\right), T_{t_{n}} g \rightarrow 0$ as $n \rightarrow \infty$ and then by using (2.20), (2.21) we obtain

$$
\int_{\mathbb{R}^{d}} g_{n}(x) h(x) d x \rightarrow 0 .
$$

Therefore, it is impossible to find convergent subsequence of $\left(g_{n}\right)_{n \in \mathbb{N}}$ in $L_{v}^{1}\left(\mathbb{R}^{d}\right)$ by $(2.32)$, (2.33) and (2.34). Thus, the embedding $I: A_{\alpha, p}^{w, \omega}\left(\mathbb{R}^{d}\right) \rightarrow L_{v}^{1}\left(\mathbb{R}^{d}\right)$ is never compact.

Again, we will give an example that the converse of the above theorem is not correct.

Example 2.9. Let $\alpha=\left(\alpha_{1}, \alpha_{2}, \ldots, \alpha_{d}\right)$, where $\alpha_{i} \neq k \pi$ for each index $i$ with $1 \leq i \leq d$ and $k \in \mathbb{Z}$. Let $w, v$ and $\omega$ be weight functions on $\mathbb{R}^{d}$ such that $v(x)=1+\|x\|$, $w(x)=(1+\|x\|)^{2}$ for all $x \in \mathbb{R}^{d}$ and $\omega$ is bounded. In that case, the embedding $I$ : 
$A_{\alpha, p}^{w, \omega}\left(\mathbb{R}^{d}\right) \rightarrow L_{v}^{1}\left(\mathbb{R}^{d}\right)$ is never compact and the function $\frac{v(x)}{w(x)}$ tends to zero as $x \rightarrow \infty$. Indeed, we get

$$
1+\|x\| \leq(1+\|x\|)^{2}
$$

for all $x \in \mathbb{R}^{d}$. Since $v \prec w$, then $A_{\alpha, p}^{w, \omega}\left(\mathbb{R}^{d}\right) \subset L_{v}^{1}\left(\mathbb{R}^{d}\right)$. Also the unit map $I: A_{\alpha, p}^{w, \omega}\left(\mathbb{R}^{d}\right) \rightarrow L_{v}^{1}\left(\mathbb{R}^{d}\right)$ is continuous. Thus by using Theorem 2.6 , it is clear that the embedding $I: A_{\alpha, p}^{w, \omega}\left(\mathbb{R}^{d}\right) \rightarrow L_{v}^{1}\left(\mathbb{R}^{d}\right)$ is never compact. On the other hand, it is easy to show that $\frac{v(x)}{w(x)}$ tends to zero as $x \rightarrow \infty$.

Theorem 2.10. Let $\alpha=\left(\alpha_{1}, \alpha_{2}, \ldots, \alpha_{d}\right)$, where $\alpha_{i} \neq k \pi$ for each index $i$ with $1 \leq i \leq d$ and $k \in \mathbb{Z}$. Let $1 \leq p<\infty$ and $w_{1}, w_{2}, \omega_{1}$ and $\omega_{2}$ be weight functions on $\mathbb{R}^{d}$. If $w_{2} \prec w_{1}$, $\omega_{2} \prec \omega_{1}$ and functions $\frac{\omega_{2}(x)}{w_{1}(x)}$ or $\frac{\omega_{2}(x)}{\omega_{1}(x)}$ do not tend to zero for $x \rightarrow \infty$, then the embedding I from $A_{\alpha, p}^{w_{1}, \omega_{1}}\left(\mathbb{R}^{d}\right)$ into $A_{\alpha, p}^{w_{2}, \omega_{2}}\left(\mathbb{R}^{d}\right)$ is not compact.

Proof. Let $w_{2} \prec w_{1}$ and $\omega_{2} \prec \omega_{1}$. Then it is known that $A_{\alpha, p}^{w_{1}, \omega_{1}}\left(\mathbb{R}^{d}\right) \subset A_{\alpha, p}^{w_{2}, \omega_{2}}\left(\mathbb{R}^{d}\right)$ by Theorem 2.5. Also there exists $c_{1}, c_{2}>0$ such that $w_{2}(x) \leq c_{1} w_{1}(x)$ and $\omega_{2}(x) \leq c_{2} \omega_{1}(x)$ for all $x \in \mathbb{R}^{d}$. Now, let us define a unit map $I$ from $A_{\alpha, p}^{w_{1}, \omega_{1}}\left(\mathbb{R}^{d}\right)$ into $A_{\alpha, p}^{w_{2}, \omega_{2}}\left(\mathbb{R}^{d}\right)$. Then we have

$$
\|I(f)\|_{A_{\alpha, p}^{w_{2}, \omega_{2}}}=\|f\|_{A_{\alpha, p}^{w_{2}, \omega_{2}}} \leq c_{1}\|f\|_{1, w_{1}}+c_{2}\left\|\mathcal{F}_{\alpha} f\right\|_{p, \omega_{1}} \leq c_{3}\|f\|_{A_{\alpha, p}^{w_{1}, \omega_{1}}}
$$

where $c_{3}=\max \left\{c_{1}, c_{2}\right\}$, for all $f \in A_{\alpha, p}^{w_{1}, \omega_{1}}\left(\mathbb{R}^{d}\right)$. So $I$ is continuous.

First of all, let the function $\frac{w_{2}(x)}{w_{1}(x)}$ does not tend to zero for $x \rightarrow \infty$. Suppose that $w_{1}(x) \rightarrow \infty$ as $x \rightarrow \infty$. Again, let us take any fixed function $g \in A_{\alpha, p}^{w_{1}, \omega_{1}}\left(\mathbb{R}^{d}\right)$. Also we take sequence $\left(t_{n}\right)_{n \in \mathbb{N}}$ in $\mathbb{R}^{d}$ such that $t_{n} \rightarrow \infty$ as $n \rightarrow \infty$. Then we define a sequence $\left(g_{n}\right)_{n \in \mathbb{N}}$ by $g_{n}=\frac{T_{t_{n}} M_{b_{n}} g}{w_{1}\left(t_{n}\right)}$, where $\left(b_{n}\right)_{n \in \mathbb{N}}$ is a sequence in $\mathbb{R}^{d}$ such that $b_{n j}=-t_{n j} \cot \alpha_{j}$. Thus, using by inequality (2.30), it is easy to see that $\left(g_{n}\right)_{n \in \mathbb{N}}$ is bounded in $A_{\alpha, p}^{w_{1}, \omega_{1}}\left(\mathbb{R}^{d}\right)$. Now, assume that there exists convergent subsequence of $\left(g_{n}\right)_{n \in \mathbb{N}}$ in $A_{\alpha, p}^{w_{2}, \omega_{2}}\left(\mathbb{R}^{d}\right)$. Since the inclusion $A_{\alpha, p}^{w_{2}, \omega_{2}}\left(\mathbb{R}^{d}\right) \subset L_{w_{2}}^{1}\left(\mathbb{R}^{d}\right)$, then the subsequence of $\left(g_{n}\right)_{n \in \mathbb{N}}$ is also convergent in $L_{w_{2}}^{1}\left(\mathbb{R}^{d}\right)$. But it is well known from the proof of Theorem 2.8 that it is impossible to obtain convergent subsequence of $\left(g_{n}\right)_{n \in \mathbb{N}}$ in $L_{w_{2}}^{1}\left(\mathbb{R}^{d}\right)$. That means the embedding $I$ is not compact. Suppose that $w_{1}$ is constant or bounded function. Similarly, let us take any fixed function $g \in C_{C}\left(\mathbb{R}^{d}\right) \cap A_{\alpha, p}^{w_{1}, \omega_{1}}\left(\mathbb{R}^{d}\right)$. Besides we get sequence $\left(t_{n}\right)_{n \in \mathbb{N}}$ in $\mathbb{R}^{d}$ such that $t_{n} \rightarrow \infty$ as $n \rightarrow \infty$. Then we define a sequence $\left(g_{n}\right)_{n \in \mathbb{N}}$ by $g_{n}=\frac{T_{t_{n}} M_{b_{n}} g}{w_{1}\left(t_{n}\right)}$, where $\left(b_{n}\right)_{n \in \mathbb{N}}$ is a sequence in $\mathbb{R}^{d}$ such that $b_{n j}=-t_{n j} \cot \alpha_{j}$. Thus, the sequence $\left(g_{n}\right)_{n \in \mathbb{N}}$ is bounded in $A_{\alpha, p}^{w_{1}, \omega_{1}}\left(\mathbb{R}^{d}\right)$ by (2.30). Now, assume that there exists convergent subsequence of $\left(g_{n}\right)_{n \in \mathbb{N}}$ in $A_{\alpha, p}^{w_{2}, \omega_{2}}\left(\mathbb{R}^{d}\right)$. Since the inclusion $A_{\alpha, p}^{w_{2}, \omega_{2}}\left(\mathbb{R}^{d}\right) \subset L_{w_{2}}^{1}\left(\mathbb{R}^{d}\right)$, then the subsequence of $\left(g_{n}\right)_{n \in \mathbb{N}}$ is also convergent in $L_{w_{2}}^{1}\left(\mathbb{R}^{d}\right)$. But it is well known from the proof of Theorem 2.8 that it is impossible to obtain convergent subsequence of $\left(g_{n}\right)_{n \in \mathbb{N}}$ in $L_{w_{2}}^{1}\left(\mathbb{R}^{d}\right)$. That means the embedding $I$ is not compact.

Now, let the function $\frac{\omega_{2}(x)}{\omega_{1}(x)}$ does not tend to zero for $x \rightarrow \infty$. Suppose that $\omega_{1}(x) \rightarrow \infty$ as $x \rightarrow \infty$. Also, let us take sequence $\left(t_{n}\right)_{n \in \mathbb{N}}$ in $\mathbb{R}^{d}$ such that $t_{n} \rightarrow \infty$ as $n \rightarrow \infty$. Thus we define a sequence $\left(u_{n}\right)_{n \in \mathbb{N}}$ in $\mathbb{R}^{d}$ such that $u_{n j}=t_{n j} \sin \alpha_{j}$. Obviously, 
$u_{n} \rightarrow \infty$ as $n \rightarrow \infty$. Now, let us take any fixed function $g \in A_{\alpha, p}^{w_{1}, \omega_{1}}\left(\mathbb{R}^{d}\right)$. Then we define a sequence $\left(g_{n}\right)_{n \in \mathbb{N}}$ by $g_{n}=\frac{M_{t_{n}} g}{\omega_{1}\left(u_{n}\right)}$. Hence, we get

$$
\left\|g_{n}\right\|_{A_{\alpha, p}^{w_{1}, \omega_{1}}}=\left\|\frac{M_{t_{n}} g}{\omega_{1}\left(u_{n}\right)}\right\|_{A_{\alpha, p}^{w_{1}, \omega_{1}}}=\frac{1}{\omega_{1}\left(u_{n}\right)}\left\|M_{t_{n}} g\right\|_{A_{\alpha, p}^{w_{1}, \omega_{1}}} .
$$

Also we have the inequality

$$
\left\|M_{x} g\right\|_{A_{\alpha, p}^{w_{1}, \omega_{1}}} \leq\|g\|_{1, w_{1}}+\omega_{1}\left(x_{1} \sin \alpha_{1}, \ldots, x_{d} \sin \alpha_{d}\right)\left\|\mathcal{F}_{\alpha} g\right\|_{p, \omega_{1}}
$$

for all $x \in \mathbb{R}^{d}$, by Theorem 5 in [19]. Thus, by using (2.35) and (2.36) we write

$$
\left\|g_{n}\right\|_{A_{\alpha, p}^{w_{1}, \omega_{1}}} \leq \frac{1}{\omega_{1}\left(u_{n}\right)}\|g\|_{1, w_{1}}+\frac{\omega_{1}\left(u_{n}\right)}{\omega_{1}\left(u_{n}\right)}\left\|\mathcal{F}_{\alpha} g\right\|_{p, \omega_{1}} \leq\|g\|_{A_{\alpha, p}^{w_{1}, \omega_{1}}} .
$$

Therefore, the sequence $\left(g_{n}\right)_{n \in \mathbb{N}}$ is bounded in $A_{\alpha, p}^{w_{1}, \omega_{1}}\left(\mathbb{R}^{d}\right)$. Now, we will show that there is no subsequence of $\left(g_{n}\right)_{n \in \mathbb{N}}$ that is convergent in $A_{\alpha, p}^{w_{2}, \omega_{2}}\left(\mathbb{R}^{d}\right)$. Let $h \in C_{C}\left(\mathbb{R}^{d}\right)$ be given. We obtain

$$
\begin{aligned}
\left|\int_{\mathbb{R}^{d}} g_{n}(x) h(x) d x\right| & \leq \frac{1}{\omega_{1}\left(u_{n}\right)} \int_{\mathbb{R}^{d}}\left|M_{t_{n}} g(x)\right||h(x)| d x \\
& \leq \frac{1}{\omega_{1}\left(u_{n}\right)}\|h\|_{\infty}\|g\|_{1} .
\end{aligned}
$$

Since $\left(u_{n}\right)_{n \in \mathbb{N}}$ and $\omega_{1}(x)$ tend to infinity as $n \rightarrow \infty$ and $x \rightarrow \infty$, respectively, we obtain

$$
\int_{\mathbb{R}^{d}} g_{n}(x) h(x) d x \rightarrow 0
$$

as $n \rightarrow \infty$. Assume that $\left(g_{n}\right)_{n \in \mathbb{N}}$ converges to $f \neq 0$ in $A_{\alpha, p}^{w_{2}, \omega_{2}}\left(\mathbb{R}^{d}\right)$. Thus we may write

$$
\left|\int_{\mathbb{R}^{d}}\left(g_{n}-f\right)(x) h(x) d x\right| \leq \int_{\mathbb{R}^{d}}\left|\left(g_{n}-f\right)(x)\right||h(x)| d x \leq\|h\|_{\infty}\left\|g_{n}-f\right\|_{A_{\alpha, p}^{w_{2}, \omega_{2}} .} .
$$

Since $\left\|g_{n}-f\right\|_{A_{\alpha, p}^{w_{2}, \omega_{2}}} \rightarrow 0$ as $n \rightarrow \infty$, then we have

$$
\int_{\mathbb{R}^{d}}\left(g_{n}-f\right)(x) h(x) d x \rightarrow 0
$$

as $n \rightarrow \infty$. Hence by using (2.37), we obtain

$$
\int_{\mathbb{R}^{d}} f(x) h(x) d x=0
$$

for all $h \in C_{C}\left(\mathbb{R}^{d}\right)$. Then $f=0$ almost everywehere. This is a contradiction. Therefore only possible limit of $\left(g_{n}\right)_{n \in \mathbb{N}}$ in $A_{\alpha, p}^{w_{2}, \omega_{2}}\left(\mathbb{R}^{d}\right)$ is zero. Let us take $a=$ $\left(x_{1} \sin \alpha_{1}, \ldots, x_{d} \sin \alpha_{d}\right)$. Thus by using Proposition 3 in [19] we write

$$
\left\|\mathcal{F}_{\alpha}\left(M_{x} g\right)\right\|_{p, \omega_{2}}=\left\|T_{a}\left(\mathcal{F}_{\alpha} g\right)\right\|_{p, \omega_{2}} .
$$

Besides, it is known by [9] that there exists $c>0$ such that

$$
c \omega_{2}(x) \leq\left\|T_{x} f\right\|_{p, \omega_{2}}
$$


for all $f \in L_{\omega_{2}}^{p}\left(\mathbb{R}^{d}\right)$ and $x \in \mathbb{R}^{d}$. Hence, by using (2.38) and (2.39) we obtain

$$
\begin{aligned}
\left\|g_{n}\right\|_{A_{\alpha, p}^{w_{2}, \omega_{2}}} & \geq \frac{1}{\omega_{1}\left(u_{n}\right)}\left\|\mathcal{F}_{\alpha}\left(M_{t_{n}} g\right)\right\|_{p, \omega_{2}} \\
& =\frac{1}{\omega_{1}\left(u_{n}\right)}\left\|T_{u_{n}}\left(\mathcal{F}_{\alpha} g\right)\right\|_{p, \omega_{2}} \\
& \geq \frac{\omega_{2}\left(u_{n}\right)}{\omega_{1}\left(u_{n}\right)} c .
\end{aligned}
$$

Since the function $\frac{\omega_{2}\left(u_{n}\right)}{\omega_{1}\left(u_{n}\right)}$ does not tend to zero for $n \rightarrow \infty$, there exists $\delta>0$ such that $\frac{\omega_{2}\left(u_{n}\right)}{\omega_{1}\left(u_{n}\right)} \geq \delta$ as $n \rightarrow \infty$. Then by using (2.40) we have

$$
\left\|g_{n}\right\|_{A_{\alpha, p}^{w_{2}, \omega_{2}}} \geq \frac{\omega_{2}\left(u_{n}\right)}{\omega_{1}\left(u_{n}\right)} c \geq c \delta .
$$

Thus, it is impossible to find convergent subsequence of $\left(g_{n}\right)_{n \in \mathbb{N}}$ in $A_{\alpha, p}^{w_{2}, \omega_{2}}\left(\mathbb{R}^{d}\right)$. Finally, the embedding $I$ is not compact. Assume that $\omega_{1}$ is constant or bounded function. Let us take any fixed function $g \in C_{C}\left(\mathbb{R}^{d}\right) \cap A_{\alpha, p}^{w_{1}, \omega_{1}}\left(\mathbb{R}^{d}\right)$, a sequence $\left(t_{n}\right)_{n \in \mathbb{N}}$ in $\mathbb{R}^{d}$ such that $t_{n} \rightarrow \infty$ as $n \rightarrow \infty$ and define a sequence $\left(g_{n}\right)_{n \in \mathbb{N}}$ by $g_{n}=M_{t_{n}} g$. Thus, using by inequality (2.18), it is easy to see that $\left(g_{n}\right)_{n \in \mathbb{N}}$ is bounded in $A_{\alpha, p}^{w_{1}, \omega_{1}}\left(\mathbb{R}^{d}\right)$. Now, assume that there exists convergent subsequence of $\left(g_{n}\right)_{n \in \mathbb{N}}$ in $A_{\alpha, p}^{w_{2}, \omega_{2}}\left(\mathbb{R}^{d}\right)$. Hence, since the inclusion $A_{\alpha, p}^{w_{2}, \omega_{2}}\left(\mathbb{R}^{d}\right) \subset L_{w_{2}}^{1}\left(\mathbb{R}^{d}\right)$, then the subsequence of $\left(g_{n}\right)_{n \in \mathbb{N}}$ is also convergent in $L_{w_{2}}^{1}\left(\mathbb{R}^{d}\right)$. But it is well known from the proof of Theorem 2.6 that it is impossible to obtain convergent subsequence of $\left(g_{n}\right)_{n \in \mathbb{N}}$ in $L_{w_{2}}^{1}\left(\mathbb{R}^{d}\right)$. That means the embedding $I$ is not compact.

We conclude the article with the following theorem, which is the main result of the paper.

Theorem 2.11. Let $\alpha=\left(\alpha_{1}, \alpha_{2}, \ldots, \alpha_{d}\right)$, where $\alpha_{i} \neq k \pi$ for each index $i$ with $1 \leq i \leq d$ and $k \in \mathbb{Z}$. Let $w_{1}, w_{2}$ and $\omega_{1}, \omega_{2}$ be weight functions on $\mathbb{R}^{d}$ and $w_{2} \prec w_{1}, \omega_{2} \prec \omega_{1}$. Then the embedding $I: A_{\alpha, 1}^{w_{1}, \omega_{1}}\left(\mathbb{R}^{d}\right) \hookrightarrow A_{\alpha, 1}^{w_{2}, \omega_{2}}\left(\mathbb{R}^{d}\right)$ is compact if and only if the functions $\frac{w_{2}(x)}{w_{1}(x)}$ and $\frac{\omega_{2}(x)}{\omega_{1}(x)}$ tend to zero as $x \rightarrow \infty$.

Proof. Let $w_{2} \prec w_{1}$ and $\omega_{2} \prec \omega_{1}$. Then it is known by Theorem 2.5 that $A_{\alpha, 1}^{w_{1}, \omega_{1}}\left(\mathbb{R}^{d}\right) \subset$ $A_{\alpha, 1}^{w_{2}, \omega_{2}}\left(\mathbb{R}^{d}\right)$. Also it is easy to see that unit map $I$ is bounded $A_{\alpha, 1}^{w_{1}, \omega_{1}}\left(\mathbb{R}^{d}\right)$ into $A_{\alpha, 1}^{w_{2}, \omega_{2}}\left(\mathbb{R}^{d}\right)$.

Now, suppose that the functions $\frac{w_{2}(x)}{w_{1}(x)}$ and $\frac{\omega_{2}(x)}{\omega_{1}(x)}$ tend to zero as $x \rightarrow \infty$. Let us take a bounded sequence $\left(f_{n}\right)_{n \in \mathbb{N}}$ in $A_{\alpha, 1}^{w_{1}, \omega_{1}}\left(\mathbb{R}^{d}\right)$. To indicate that the embedding $A_{\alpha, 1}^{w_{1}, \omega_{1}}\left(\mathbb{R}^{d}\right) \hookrightarrow A_{\alpha, 1}^{w_{2}, \omega_{2}}\left(\mathbb{R}^{d}\right)$ is compact, we will prove that $\left(f_{n}\right)$ has a convergent subsequence in $A_{\alpha, 1}^{w_{2}, \omega_{2}}\left(\mathbb{R}^{d}\right)$. As the sequence $\left(f_{n}\right)_{n \in \mathbb{N}}$ is bounded in $A_{\alpha, 1}^{w_{1}, \omega_{1}}\left(\mathbb{R}^{d}\right)$, there exists $c_{1}>0$ such that

$$
\left\|f_{n}\right\|_{A_{\alpha, 1}^{w_{1}, \omega_{1}}} \leq c_{1}
$$

for all $n \in \mathbb{N}$. Moreover, boundedness of the unit map $I$, there exists $c_{2}>0$ such that

$$
\left\|I\left(f_{n}\right)\right\|_{A_{\alpha, 1}^{w_{2}, \omega_{2}}}=\left\|f_{n}\right\|_{A_{\alpha, 1}^{w_{2}, \omega_{2}}} \leq c_{2}\left\|f_{n}\right\|_{A_{\alpha, 1}^{w_{1}, \omega_{1}}} .
$$


Since, the functions $\frac{w_{2}(x)}{w_{1}(x)}, \frac{\omega_{2}(x)}{\omega_{1}(x)}$ tend to zero as $x \rightarrow \infty$, then there exist $\delta_{k}, \delta_{k}^{\prime}>0$ such that

$$
\frac{w_{2}(x)}{w_{1}(x)}<\frac{1}{k}, \quad \text { and } \quad \frac{\omega_{2}(x)}{\omega_{1}(x)}<\frac{1}{k}
$$

where $\|x\|>\delta_{k}$ and $\|x\|>\delta_{k}^{\prime}$, respectively for any $k \in \mathbb{N}$. Thus there are sequences of increasing balls $\left(B_{\delta_{k}}\right)_{k \in \mathbb{N}}$ and $\left(B_{\delta^{\prime} k}\right)_{k \in \mathbb{N}}$ which are centered at origin and $\delta_{k} \rightarrow \infty$, $\delta_{k}^{\prime} \rightarrow \infty$ as $k \rightarrow \infty$ such that providing the inequalities (2.43), where $x \in \mathbb{R}^{d}-B_{\delta_{k}}$ and $x \in \mathbb{R}^{d}-B_{\delta^{\prime} k}$, respectively for any $k \in \mathbb{N}$. Let $S_{k}=\left(B_{\delta_{k}} \cup B_{\delta^{\prime}{ }_{k}}\right)$. Then we can write the inequalities (2.43) such that $x \in \mathbb{R}^{d}-S_{k}$ for $k \in \mathbb{N}$.

Suppose that, $\left(t_{n}\right)_{n \in \mathbb{N}}$ be any sequence which is dense in $S_{1}$. Hence by (2.42), we get

$$
\begin{aligned}
\left\|\mathcal{F}_{\alpha} f_{n}\right\|_{\infty} & =\sup _{u \in \mathbb{R}^{d}}\left|\prod_{j=1}^{d} \sqrt{\frac{1-i \cot \alpha_{j}}{2 \pi}} \int_{\mathbb{R}^{d}} f_{n}(t) e^{\sum_{j=1}^{d}\left(\frac{i}{2}\left(u_{j}^{2}+t_{j}^{2}\right) \cot \alpha_{j}-i u_{j} t_{j} \operatorname{cosec} \alpha_{j}\right)} d t\right| \\
& \leq \prod_{j=1}^{d}\left|\sqrt{\frac{1-i \cot \alpha_{j}}{2 \pi}}\right| \int_{\mathbb{R}^{d}}\left|f_{n}(t)\right| d t \\
& \leq \prod_{j=1}^{d}\left|\sqrt{\frac{1-i \cot \alpha_{j}}{2 \pi}}\right|\left\|f_{n}\right\|_{A_{\alpha, 1}^{w_{2}, \omega_{2}}} \\
& \leq \prod_{j=1}^{d}\left|\sqrt{\frac{1-i \cot \alpha_{j}}{2 \pi}}\right| c_{2}\left\|f_{n}\right\|_{A_{\alpha, 1}^{w_{1}, \omega_{1}}}
\end{aligned}
$$

Also, let $c_{0}=\prod_{j=1}^{d}\left|\sqrt{\frac{1-i \cot \alpha_{j}}{2 \pi}}\right| c_{1} c_{2}$. Then by using inequality (2.41), we obtain

$$
\left\|\mathcal{F}_{\alpha} f_{n}\right\|_{\infty} \leq c_{0}
$$

Therefore there exists a subsequence $\left(f_{n_{1}(k)}\right)_{n_{1}(k) \in \mathbb{N}}$ of $\left(f_{n}\right)_{n \in \mathbb{N}}$ such that the sequence $\left(\mathcal{F}_{\alpha} f_{n_{1}(k)}\left(t_{1}\right)\right)_{n_{1}(k) \in \mathbb{N}}$ converges. Likewise, there exists a subsequence $\left(f_{n_{2}(k)}\right)_{n_{2}(k) \in \mathbb{N}}$ of $\left(f_{n_{1}(k)}\right)_{n_{1}(k) \in \mathbb{N}}$ such that the sequence $\left(\mathcal{F}_{\alpha} f_{n_{2}(k)}\left(t_{2}\right)\right)_{n_{2}(k) \in \mathbb{N}}$ converges. Continuing this method, there exists a subsequence $\left(f_{n_{l}(k)}\right)_{n_{l}(k) \in \mathbb{N}}$ of $\left(f_{n_{l-1}(k)}\right)_{n_{l-1}(k) \in \mathbb{N}}$ such that the sequence $\left(\mathcal{F}_{\alpha} f_{n_{l}(k)}\left(t_{l}\right)\right)_{n_{l}(k) \in \mathbb{N}}$ converges. Hence the sequence $\left(\mathcal{F}_{\alpha} f_{n_{l}(k)}\left(t_{r}\right)\right)_{n_{l}(k) \in \mathbb{N}}$ converges, for all $l \in \mathbb{N}$ such that $r \leq l$. Let $m(k)=n_{k}(k)$, then the sequence $\left(\mathcal{F}_{\alpha} f_{m(k)}\left(t_{l}\right)\right)_{m(k) \in \mathbb{N}}$ converges which is subsequence of $\left(\mathcal{F}_{\alpha} f_{n_{l}(k)}\left(t_{l}\right)\right)_{n_{l}(k) \in \mathbb{N}}$. Thus there exists a subsequence $\left(g_{m}\right)_{m \in \mathbb{N}}$ of $\left(f_{n}\right)_{n \in \mathbb{N}}$ such that the sequence $\left(\mathcal{F}_{\alpha} g_{m}\left(t_{l}\right)\right)_{m \in \mathbb{N}}$ converges, for all $l \in \mathbb{N}$. Also, since the sequence $\left(t_{n}\right)_{n \in \mathbb{N}}$ is dense in $S_{1}$, then the sequence $\left(\mathcal{F}_{\alpha} g_{m}(x)\right)_{m \in \mathbb{N}}$ converges, for any $x \in S_{1}$. Using the method reviewed above, we can find a sequence $\left(u_{m}\right)_{m \in \mathbb{N}}$ which is a subsequence of $\left(g_{m}\right)_{m \in \mathbb{N}}$ such that the sequence $\left(\mathcal{F}_{\alpha} u_{m}(x)\right)_{m \in \mathbb{N}}$ converges, for all $x \in S_{2}$. Reiterating this technique, we obtain a sequence $\left(h_{n}\right)_{n \in \mathbb{N}}$ which is a subsequence of $\left(f_{n}\right)_{n \in \mathbb{N}}$ such that the sequence $\left(\mathcal{F}_{\alpha} h_{n}(x)\right)_{n \in \mathbb{N}}$ converges, for all $x \in S_{k}$. Also, let $\beta=\left(\beta_{1}, \beta_{2}, \ldots, \beta_{d}\right)$, such that $\alpha=\left(\alpha_{1}, \alpha_{2}, \ldots, \alpha_{d}\right)$, where $\beta_{i}+\alpha_{i}=2 \pi$ for each index $i$ with $1 \leq i \leq d$ and $k \in \mathbb{Z}$, and $\prod_{j=1}^{d}\left|\sqrt{\frac{1-i \cot \beta_{j}}{2 \pi}}\right|=M_{1}$. Then by using (2.41) and (2.42) we get

$$
\begin{aligned}
\left\|h_{n}\right\|_{\infty} & =\left\|\mathcal{F}_{\beta}\left(\mathcal{F}_{\alpha} h_{n}\right)\right\|_{\infty} \leq \prod_{j=1}^{d}\left|\sqrt{\frac{1-i \cot \beta_{j}}{2 \pi}}\right|\left\|\mathcal{F}_{\alpha} h_{n}\right\|_{1} \\
& \leq M_{1} c_{2}\left\|h_{n}\right\|_{A_{\alpha, 1}^{w_{1}, \omega_{1}} \leq M_{1} c_{2} c_{1} .}
\end{aligned}
$$


Hence, $\left(h_{n}\right)_{n \in \mathbb{N}}$ is bounded. By applying the steps in the first part of this proof, we have a sequence $\left(s_{n}\right)_{n \in \mathbb{N}}$ that is a subsequence of $\left(h_{n}\right)_{n \in \mathbb{N}}$, where the sequence $\left(s_{n}\right)_{n \in \mathbb{N}}$ converges on $S_{k}$, for all $k \in \mathbb{N}$.

Furthermore, by (2.41) and (2.43) we have

$$
\begin{aligned}
& \left\|s_{n}-s_{m}\right\|_{A_{\alpha, 1}^{w_{2}, \omega_{2}}} \\
& =\left\|s_{n}-s_{m}\right\|_{1, w_{2}}+\left\|\mathcal{F}_{\alpha}\left(s_{n}-s_{m}\right)\right\|_{1, \omega_{2}} \\
& =\int_{S_{k}}\left|s_{n}(x)-s_{m}(x)\right| w_{2}(x) d x+\int_{\mathbb{R}^{d}-S_{k}}\left|s_{n}(x)-s_{m}(x)\right| w_{2}(x) d x \\
& \quad+\int_{S_{k}}\left|\mathcal{F}_{\alpha} s_{n}(x)-\mathcal{F}_{\alpha} s_{m}(x)\right| \omega_{2}(x) d x+\int_{\mathbb{R}^{d}-S_{k}}\left|\mathcal{F}_{\alpha} s_{n}(x)-\mathcal{F}_{\alpha} s_{m}(x)\right| \omega_{2}(x) d x \\
& \leq \int_{S_{k}}\left|s_{n}(x)-s_{m}(x)\right| w_{2}(x) d x+\int_{S_{k}}\left|\mathcal{F}_{\alpha} s_{n}(x)-\mathcal{F}_{\alpha} s_{m}(x)\right| \omega_{2}(x) d x \\
& \quad+\frac{1}{k}\left(\left\|s_{n}\right\|_{1, w_{1}}+\left\|s_{m}\right\|_{1, w_{1}}\right)+\frac{1}{k}\left(\left\|\mathcal{F}_{\alpha} s_{n}\right\|_{1, \omega_{1}}+\left\|\mathcal{F}_{\alpha} s_{m}\right\|_{1, \omega_{1}}\right) \\
& \leq \int_{S_{k}}\left|s_{n}(x)-s_{m}(x)\right| w_{2}(x) d x+\int_{S_{k}}\left|\mathcal{F}_{\alpha} s_{n}(x)-\mathcal{F}_{\alpha} s_{m}(x)\right| \omega_{2}(x) d x+\frac{4 c_{1}}{k} .
\end{aligned}
$$

Let $\varepsilon>0$ be given. Also, we select $k$ large enough where $\frac{4 c_{1}}{k}<\frac{\varepsilon}{3}$. Besides the sequences $\left(s_{n}\right)_{n \in \mathbb{N}}$ and $\left(\mathcal{F}_{\alpha} s_{n}\right)_{n \in \mathbb{N}}$ converge on $\overline{S_{k}}$ such that the set $\overline{S_{k}}$ is closure of $S_{k}$. Since the sequences $\left(s_{n}\right)_{n \in \mathbb{N}}$ and $\left(\mathcal{F}_{\alpha} s_{n}\right)_{n \in \mathbb{N}}$ converge pointwise on $\overline{S_{k}}$, and according to the inequalties (2.44) and (2.45) and the bounded converge theorem there exists $n_{1} \in \mathbb{N}$ such that

$$
\int_{S_{k}}\left|s_{n}(x)-s_{m}(x)\right| w_{2}(x) d x<\frac{\varepsilon}{3}
$$

for all $m, n \geq n_{1}$ and also there exists $n_{2} \in \mathbb{N}$ such that

$$
\int_{S_{k}}\left|\mathcal{F}_{\alpha} s_{n}(x)-\mathcal{F}_{\alpha} s_{m}(x)\right| \omega_{2}(x) d x<\frac{\varepsilon}{3}
$$

for all $m, n \geq n_{2}$. Let $N=\max \left\{n_{1}, n_{2}\right\}$. Thus, combining (2.46), (2.47) and (2.48) we get

$$
\begin{aligned}
\left\|s_{n}-s_{m}\right\|_{A_{\alpha, 1}^{w_{2}, \omega_{2}}} & \leq \int_{S_{k}}\left|s_{n}(x)-s_{m}(x)\right| w_{2}(x) d x \\
& +\int_{S_{k}}\left|\mathcal{F}_{\alpha} s_{n}(x)-\mathcal{F}_{\alpha} s_{m}(x)\right| \omega_{2}(x) d x+\frac{4 c_{1}}{k}<\varepsilon
\end{aligned}
$$

for all $m, n \geq N$. This implies that the sequence $\left(s_{n}\right)_{n \in \mathbb{N}}$ is a Cauchy sequence in $A_{\alpha, 1}^{w_{2}, \omega_{2}}\left(\mathbb{R}^{d}\right)$. Thus, the embedding of the space $A_{\alpha, 1}^{w_{1}, \omega_{1}}\left(\mathbb{R}^{d}\right)$ into $A_{\alpha, 1}^{w_{2}, \omega_{2}}\left(\mathbb{R}^{d}\right)$ is compact.

Conversely, if the functions $\frac{w_{2}(x)}{w_{1}(x)}$ or $\frac{\omega_{2}(x)}{\omega_{1}(x)}$ do not tend to zero for $x \rightarrow \infty$, then by Theorem 2.10 the embedding of the space $A_{\alpha, 1}^{w_{1}, \omega_{1}}\left(\mathbb{R}^{d}\right)$ into $A_{\alpha, 1}^{w_{2}, \omega_{2}}\left(\mathbb{R}^{d}\right)$ is not compact. This is the desired result.

Acknowledgment. The authors would like to thank the reviewer for his/her valuable comments and constructive suggestions

\section{References}

[1] T. Alieva, V. Lopez, F. Agullo-Lopez and L.B. Almeida, The fractional Fourier transform in optical propagation problems, J. Modern Opt. 41 (5), 1037-1044, 1994.

[2] L.B. Almeida, The fractional Fourier transform and time-frequency representations, IEEE Trans. Signal Process. 42 (11), 3084-3091, 1994.

[3] L.B. Almeida, Product and convolution theorems for the fractional Fourier transform, IEEE Signal Process. Lett. 4 (1), 15-17, 1997. 
[4] A. Bultheel and H. Martinez, A shattered survey of the fractional Fourier transform, K.U.Leuven: Department of Computer Science, Report TW337, 2002.

[5] P. Dimovski , S. Pilipovic, B. Prangoski and J. Vindas, Translation-modulation invariant Banach spaces of ultradistributions, J. Fourier Anal. Appl. 25 (3), 819-841, 2019.

[6] R.G. Dorsch, A.W. Lohmann, Y. Bitran, D. Mendlovic and H.M. Ozaktas, Chirp filtering in the fractional Fourier domain, Appl. Optics, 33 (32), 7599-7602, 1994.

[7] H.G. Feichtinger, A compactness criterion for translation invariant Banach spaces of functions, Anal. Math. 8, 165-172, 1982.

[8] H.G. Feichtinger, Compactness in translation invariant Banach spaces of distributions and compact multipliers, J. Math. Anal. Appl. 102, 289-327, 1984.

[9] H.G. Feichtinger and A.T. Gürkanlı, On a family of weighted convolution algebras, Int. J. Math. Sci. 13 (3), 517-526, 1990.

[10] R.H. Fischer, A.T. Gürkanlı and T.S. Liu, On a family of weighted spaces, Math. Slovaca, 46 (1), 71-82, 1996.

[11] K. Gröchenig, Weight functions in time-frequency analysis, in: Pseudodifferential Operators: Partial Differential Equations and Time-Frequency Analysis, Fields Inst. Commun. 52, 343-366, Amer. Math. Soc., Providence, RI, 2007.

[12] A.T. Gürkanl, Compact embeddings of spaces $A_{w, \omega}^{p}\left(\mathbb{R}^{d}\right)$, Taiwanese J. Math. $12(7)$, 1757-1767, 2008.

[13] A.C. McBride and F.H. Kerr, On Namias's fractional Fourier transforms, IMA J. Appl. Math. 39, 159-175, 1987.

[14] V. Namias, The fractional order of Fourier transform and its application in quantum mechanics, J. Inst. Math. Appl. 25, 241-265, 1980.

[15] H.M. Ozaktas, M.A. Kutay and Z. Zalevsky, The Fractional Fourier Transform with Applications in Optics and Signal Processing, Chichester, John Wiley and Sons, 2001.

[16] H. Reiter, Classical Harmonic Analysis and Locally Compact Group, Oxford University Press, 1968.

[17] W. Rudin, Real and Complex Analysis, New York, McGraw-Hill, 1966.

[18] W. Rudin, Functional Analysis, McGraw-Hill, 1973.

[19] E. Toksoy and A. Sandıkçı, On function spaces with fractional Fourier transform in weighted Lebesgue spaces, J. Inequal. Appl. 2015 (1), Article Id:87, 1-10, 2015.

[20] N. Wiener, Hermitian polynomials and Fourier analysis, J. Math. Phys. 8, 70-73, 1929.

[21] A.I. Zayed, On the relationship between the Fourier and fractional Fourier transforms, IEEE Signal Proc. Let. 3, 310-311, 1996. 